\title{
Structural studies of arginine decarboxylase in Helicobacter pylori
}

\author{
Huawei $Z \mathrm{HANG}^{1}$, Shannon Wing Ngor $\mathrm{AU}^{1}$ \\ ${ }^{1}$ School of Life Sciences, The Chinese University of Hong Kong, Hong Kong.
}

Arginine decarboxylase (ADC) hydrolyzes L-arginine into agmatine and carbon dioxide and is the key enzyme in polyamine biosynthesis pathway. It was reported that ADC confers bacteria acidic resistance through $\mathrm{pH}$-dependent oligomerization and thus helps bacterial pathogens survive in acidic environment. This is particularly important for human pathogen Helicobacter pylori which colonizes in human stomach and causes various diseases like gastritis and gastric cancer. Previous studies show that ADC involves in acid tolerance of $H$. pylori but the underlining mechanism is not clear. In this study, we will combine X-ray crystallography, cryo-EM, analytical ultracentrifugation and other biochemical methods to determine the oligomerization and atomic structures of $H$. pylori ADC under different $\mathrm{pH}$. The key residues for the conformational changes will be identified and confirmed by mutagenesis. The enzymic activity under different $\mathrm{pH}$ will also be measured. Our studies will provide further understanding on the mechanism of acid resistance in $\mathrm{H}$. pylori. 